\title{
Etiología de la neumonía asociada a ventilación mecánica en un hospital clínico. Asociación con co-morbilidad, uso previo de antimicrobianos y mortalidad
}

\author{
Mauricio Ruiz C., Julia Guerrero P. y Carlos Romero P.
}

\section{Etiology of ventilator-associated pneumonia in a university hospital. Association with comorbidity, previous use of antibiotics and mortality}

Ventilator-associated pneumonia (VAP) is a complication with an increased risk of morbidity and mortality. Inadequate antibiotic treatment is a risk factor of mortality which can be improved. For this reason it is important to know the local etiology of VAP. During a one year-period we investigated the etiology of VAP in a teaching hospital. Forty eight VAP were included, of which 19 were women. The median age was 59.5 (range 17-91 years), twelve VAP were early onset. Methicillin resistant Staphylococcus aureus (MRSA) was the main microorganism isolated, regardless of timing of diagnosis of VAP, followed by polimicrobial etiology, Acinetobacter sp and $P$. aeruginosa. Etiology was not associated with comorbidity; however previous antibiotic use was related with MRSA and polymicrobial etiology. Mortality was $35 \%$ and was mainly associated with $P$. aeruginosa isolation. Conclusion: MRSA was the main cause of VAP regardless of the timing of its occurrence.

Key words: Ventilator-associated pneumonia; Etiology; Intensive Care Unit; Staphylococcus aureus; Methicillin resistance.

Palabras claves: Neumonía asociada a la ventilación mecánica; Etiología; Unidad de Cuidados Intensivos; Staphylococcus aureus; Resistencia a meticilina.

\section{Introducción}

$\mathrm{L}$ a neumonía asociada a la ventilación mecánica (NAVM) es una complicación que afecta a aproximadamente entre 9 y $27 \%$ de los pacientes sometidos a este soporte por más de 48 horas. El riesgo de neumonía es mayor los primeros días de ventilación mecánica (VM), con una incidencia de 3\% diario los primeros cinco días, 2\% diario hasta el décimo día y $1 \%$ los días posteriores ${ }^{1}$. Dicha frecuencia se eleva en situaciones particularmente graves llegando hasta $70 \%$ en grandes quemados o en asociación con distress respiratorio agudo ${ }^{2}$.

La letalidad atribuida a esta complicación fluctúa entre 24 y $76 \%{ }^{2}$ y su morbilidad medida como prolongación de la estadía en la unidad de cuidados intensivos (UCI) se incrementa significativamente. Por ejemplo, Jiménez et al, en una UCI polivalente, describió una prolongación promedio de la VM en 22 días $^{3}$. Asimismo, el rango de días extras en la UCI atribuible a la NAVM, es entre 15 y 43 días $^{2}$. No menos importante es el aumento en los costos derivados de esta complicación, que puede llegar a US\$ 40.000 por episodio ${ }^{4}$.
Diversos intentos destinados a reducir el impacto de la NAVM en la morbilidad y letalidad de los pacientes críticos han fracasado. Explicaciones para este hecho son diversas, algunas inherentes al progreso de la medicina que aumenta la co-morbilidad y gravedad de los pacientes admitidos en la UCI. No obstante, un factor importante que puede ser modificado es el tratamiento antimicrobiano empírico inadecuado, esto es, que no cubra, al menos, un microorganismo responsable de la neumonía o que éste sea resistente.

Como es sabido, ante la sospecha clínica de una NAVM es imperativo tomar cultivos e iniciar un tratamiento antimicrobiano empírico ya que el retraso en su inicio también incrementa su mortalidad5; cuando el esquema seleccionado no es adecuado, el riesgo relativo se incrementa significativamente ${ }^{6}$. Por este motivo, se han hecho esfuerzos para estandarizar el tratamiento antimicrobiano como una forma de reducir las indicaciones inadecuadas, lo que se ha plasmado en numerosas directrices, una de ellas, nacional ${ }^{7}$.

Lamentablemente, y como quedó establecido en las pautas chilenas para el tratamiento de la NAVM, no hay estudios nacionales que hayan investigado la
Hospital Clínico Universidad de

Chile, Santiago-Chile

Sección de Enfermedades Respiratorias (MRC)

Unidad de Pacientes Críticos (JGP, CRP)

Recibido: 24 mayo 2006

Aceptado: 14 octubre 2006

Correspondencia a:

Mauricio Ruiz Carmona mruiz@redclinicauchile.cl 
etiología de esta infección nosocomial, y por tanto, las recomendaciones realizadas sólo se basaron en experiencias extranjeras. Por este motivo, decidimos realizar un estudio prospectivo para establecer la etiología de la NAVM en nuestro hospital y correlacionarla con co-morbilidad, gravedad al ingreso a la UCI y mortalidad.

\section{Pacientes y Métodos}

Durante el período comprendido entre julio del 2004 y julio del 2005 se estudiaron todas las neumonías con confirmación microbiológica observadas en pacientes ventilados mecánicamente por más de 48 horas, en una UCI de adultos polivalente con 12 cupos, de un hospital universitario de 600 camas. Todo paciente que en el curso de su evolución presentó infiltrados radiológicos nuevos o progresivos en la radiografía de tórax portátil más al menos dos de los siguientes criterios:

- Temperatura axilar $>38^{\circ} \mathrm{Co}<35^{\circ} \mathrm{C}$

- Leucocitosis $>10.000 / \mathrm{mm}^{3} \mathrm{o}<4.000 / \mathrm{mm}^{3}$

- Secreciones traqueales purulentas ${ }^{8}$, fue considerado sospechoso de cursar con NAVM y se le efectuó cultivo de la vía aérea.

Obtención de muestras para estudio microbiológico. Las técnicas utilizadas fueron: cultivo cuantitativo de aspirado traqueal (CCAT), lavado broncoalveolar (LBA) a ciegas o a través del fibrobroncoscopio; la técnica utilizada fue decisión del equipo de médicos intensivistas de acuerdo a la factibilidad técnica. La muestra para CCAT se obtuvo utilizando un catéter de aspiración introducido por el tubo endotraqueal y conectando el otro extremo del catéter a un colector estéril; el catéter de aspiración se introducía hasta encontrar resistencia y en ese punto se conectaba a la aspiración hasta obtener material para cultivo. Nunca se instiló solución salina fisiológica para evitar falsos negativos. La técnica utilizada para el LBA a ciegas consistió en una sonda nasogástrica número 14 cortada, con tijeras estériles, proximal a las fenestraciones. La sonda era introducida por el tubo endotraqueal hasta encontrar resistencia y luego, por el otro extremo se instiló $50 \mathrm{ml}$ de solución salina fisiológica estéril a través de una jeringa con punta tipo "Tumi", para luego aspirar suavemente. El líquido obtenido se recolectó en un frasco de boca ancha y con tapa para su procesamiento microbiológico. Para el LBA vía fibrobroncoscópica, el ventilador mecánico fue programado con una $\mathrm{FiO}_{2}$ de $100 \%$. Posteriormente, se sedaba al paciente con una mezcla de midazolam y fentanilo, para luego administrar un relajante muscular no depolarizante. Se introducía sin aspirar el fibrobroncoscopio marca Olympus BF1T30 hasta un bronquio subsegmentario que tenía secreción purulenta y que coincidía con el infiltrado radiológico observado. Enseguida se instilaba $120 \mathrm{ml}$ de solución salina fisiológica estéril, los primeros $20 \mathrm{ml}$ instilados eran descartados y el resto se recolectaba en un frasco de boca ancha y con tapa, para su procesamiento microbiológico.

Técnicas microbiológicas. El procesamiento de los cultivos cuantitativos fue el siguiente: Para el CCAT se diluyó en proporción 1:2 con solución salina fisiológica estéril, luego se homogenizó por dos minutos con perlas de vidrio estériles. Una vez homogenizado se extrajo $100 \mu \mathrm{L}$ y se diluyó $1: 100$ con solución salina fisiológica estéril y se agitó en vortex. Se rotuló una placa de agar sangre y una de agar Mc Conkey como placas A y otro set como placas B. Posteriormente, se sembró $100 \mu \mathrm{L}$ de la muestra diluida en cada placa $\mathrm{A}$, diseminando por medio de bagueta de vidrio doblada (dilución final placas A: 1 en 2.000). En forma análoga se inoculó $10 \mu \mathrm{L}$ de la muestra diluida en cada placa B (dilución final placas B: 1 en 20.000). Las placas fueron incubadas en atmósfera aeróbica a $35^{\circ} \mathrm{C}$ hasta 72 horas. De acuerdo con esta técnica, cada colonia presente en las placas A equivale a $2.000 \mathrm{ufc} / \mathrm{ml}$ y cada colonia de las placas B a $20.000 \mathrm{ufc} / \mathrm{ml}^{7}$. Independientemente de la forma en que se obtuvo el LBA, el procesamiento microbiológico fue el mismo y consistió en homogeneizar la muestra en el vórtex durante tres minutos para luego agregar $10 \mu \mathrm{L}$ de la muestra en cada uno de los siguientes medios: agar sangre (en atmósfera con $\mathrm{CO}_{2}$ ), agar Mc Conkey, agar chocolate equino (en atmósfera con $\mathrm{CO}_{2}$ ), agar Sabouraud, y se incubó a $35^{\circ} \mathrm{C}$. El número de colonias obtenidas se multiplicaba por 100 (factor de dilución). Los niveles de corte considerados significativos para establecer una relación etiológica con la NAVM fueron $10^{6} \mathrm{ufc} /$ $\mathrm{ml}, 10^{3} \mathrm{ufc} / \mathrm{ml}$ y $10^{4} \mathrm{ufc} / \mathrm{ml}$ para CCAT, LBA a ciegas y LBA fibrobroncoscópico, respectivamente.

Criterios de inclusión y exclusión. Sólo los pacientes con cultivo positivo de la vía aérea fueron incluidos en el estudio. Fueron excluidos pacientes portadores conocidos de VIH, en uso de prednisona durante un mes o más, a dosis mayores de $20 \mathrm{mg} / \mathrm{día}$, en inmunosupresión previa al ingreso a la UCI (trasplantados, usuarios de tratamiento inmunosupresor o quimioterapia).

Sólo un episodio por paciente fue incluido. Se consideró como NAVM precoz si ésta ocurría dentro de los primeros cuatro días de ingreso a la UCI, en caso contrario se clasificó como tardía.

A todo paciente incluido en el protocolo se le registraron datos bio-demográficos (edad y sexo), presencia de co-morbilidad definida como enfermedad(es) crónica(s) concomitante(s) a la NAVM, motivo de in- 
greso a la UCI, APACHE II (acute physiology and chronic health evaluation) al ingreso y diagnóstico de neumonía, tipo de cultivo realizado de la vía aérea, resultado microbiológico, tratamiento antimicrobiano empírico recibido y seguimiento evolutivo por 30 días.

Finalmente, se evaluó la letalidad de la serie a 30 días, tratamiento antimicrobiano inadecuado y la presencia de patógenos multiresistentes. Esto último se definió como la presencia de Staphylococcus aureus meticilina resistente (SAMR), Acinetobacter sp, Pseudomonas aeruginosa resistentes a cefalosporinas de tercera generación anti-pseudomónicas y enterobacterias productoras de $\beta$-lactamasas de espectro extendido.

El estudio fue aprobado por el comité de ética institucional.

Análisis estadístico. Variables continuas fueron comparadas con test $t$ de Student. Variables categóricas fueron comparadas usando el test del $\chi^{2}$. Las variables clínicas, co-morbilidad y mortalidad fueron correlacionadas con la etiología en un análisis univariado, todas aquellas asociaciones con $\mathrm{p}<0,10$ fueron sometidos a un análisis multivariado. Se consideró estadísticamente significativo con una $\mathrm{p}<0,05$. Para el análisis estadístico se utilizó el programa SPSS versión 12.0

\section{Resultados}

Durante el período de estudio se recopilaron 48 casos de NAVM con demostración etiológica, 19 pacientes fueron mujeres, la mediana de edad fue 59,5 años (rango 17 a 91), 12 de ellas fueron de instalación precoz. El APACHE II de ingreso a UCI fue de $21 \pm 6$.

En la Tabla 1 se detallan las características biodemográficas y la co-morbilidad de la serie; en ella se puede observar, como es lógico, un mayor tiempo transcurrido al diagnóstico de NAVM para la tardía; no obstante, llama la atención que para la neumonía precoz, hay una importante diferencia entre las horas transcurridas desde la hospitalización y el ingreso a la UCI o conexión a la ventilación mecánica.

La presencia de co-morbilidad fue un hecho frecuente de la serie, en particular la neurológica en la neumopatía precoz y diabetes mellitus tipo 2 en la forma tardía. Sólo un paciente fue derivado de otro centro. La técnica microbiológica más utilizada fue el CCAT, el que dio el diagnóstico en 47 ocasiones. En dos oportunidades se realizó en forma simultánea un LBA a ciegas cuyo resultado fue concordante con el CCAT y en un solo caso se hizo un LBA vía fibrobroncoscópica.
La mayoría de los pacientes (46/48) estaba recibiendo antimicrobianos al momento de la sospecha clínica de NAVM. Los resultados microbiológicos se detallan en la Tabla 2; en ella se aprecia que la principal causa de NAVM fue SAMR, seguido por la etiología polimicrobiana. En este último grupo, en todos salvo un caso, SAMR fue uno de los agentes involucrados.

Tabla 1. Características bio-demográficas y co-morbilidad de la serie

\begin{tabular}{lccl} 
Factor & $\begin{array}{c}\text { Precoz } \\
(\mathbf{n : ~ 1 2 )}\end{array}$ & $\begin{array}{c}\text { Tardía } \\
(\mathbf{n : ~ 3 6 )}\end{array}$ & $\mathbf{p}$ \\
\hline Sexo (M/H) & $6 / 6$ & $13 / 23$ & 0,3 \\
\hline Edad/años (M) & $60,5(22-91)$ & $58,3(17-87)$ & 0,8 \\
\hline Horas de hospitalización previas a NAVM & $203 \pm 274,2$ & $267,4 \pm 140,4$ & 0,04 \\
\hline Horas de UCl previas a NAVM & $72,9 \pm 17,5$ & $228,4 \pm 137,3$ & 0,014 \\
\hline Horas de VM previas a NAVM & $69,7 \pm 18,7$ & $223,5 \pm 127,6$ & 0,015 \\
APACHE II al ingreso & $20,6 \pm 5,5$ & $21,2 \pm 6$ & 0,9 \\
\hline Antimicrobiano previo (S/N) & $11 / 1$ & $35 / 1$ & 0,1 \\
Co-morbilidad (S/N) & $11 / 1$ & $28 / 8$ & 0,02 \\
$\quad$ Neurológica (S/N) & $6 / 6$ & $8 / 28$ & 0,03 \\
Cardiovascular (S/N) & $3 / 9$ & $12 / 24$ & 0,2 \\
Pulmonar (S/N) & $0 / 12$ & $3 / 33$ & 0,03 \\
Daño hepático crónico /S/N) & $2 / 10$ & $9 / 27$ & 0,2 \\
Diabetes mellitus (S/N) & $0 / 12$ & $6 / 30$ & 0,000 \\
Insuficiencia renal crónica (S/N) & $0 / 12$ & $2 / 34$ & 0,087
\end{tabular}

APACHE II: Acute physiology and chronic health evaluation. Co-morbilidad neurológica: Parkinson, AVE secuelado, demencia. Co-morbilidad cardiovascular: cardiopatía coronaria, insuficiencia cardíaca. Co-morbilidad respiratoria: EPOC, asma, bronquiectasias, secuelas de TBC. Insuficiencia renal crónica: creatininemia $>2 \mathrm{mg} / \mathrm{dl}$

Tabla 2. Especies aisladas según tiempo de aparición de la neumonía asociada a ventilación mecánica

\begin{tabular}{|c|c|c|}
\hline Microorganismo & $\begin{array}{c}\text { Precoz } \\
\text { n: } 12 \\
(\%)\end{array}$ & $\begin{array}{c}\text { Tardía } \\
\text { n: } 36 \\
(\%)\end{array}$ \\
\hline Pseudomonas aeruginosa & $1 \quad(8)$ & $8 \quad(22)$ \\
\hline SAMR & $7 \quad(58)$ & 19 (53) \\
\hline SAMS & $1 \quad(8)$ & $0 \quad(0)$ \\
\hline Acinetobacter sp & $3 \quad(25)$ & 13 (36) \\
\hline Enterobacter sp & $2(17)$ & $7 \quad(19)$ \\
\hline Stenotrophomonas maltophilia & $0 \quad(0)$ & $1 \quad(3)$ \\
\hline Haemophilus influenzae & $2(17)$ & 0 \\
\hline Enterococcus sp & $3 \quad(25)$ & $1 \quad$ (3) \\
\hline Cultivo polimicrobiano & $6 \quad(50)$ & $10 \quad(28)$ \\
\hline Total microorganismos & 18 & 53 \\
\hline
\end{tabular}

SAMR: Staphylococcus aureus meticilina resistente; SAMS: Staphylococcus aureus meticilina sensible 


\begin{tabular}{|c|c|c|c|c|c|c|c|c|}
\hline Etiología & Precoz/tardía & $\mathbf{p}$ & $\begin{array}{l}\text { Antimicrobiano } \\
\text { previo }(s / n)\end{array}$ & $\mathbf{P}$ & $\begin{array}{l}\text { Antimicrobiano } \\
\text { inadecuado }(s / n)\end{array}$ & $\mathbf{p}$ & Letalidad (s/n) & $\mathbf{p}$ \\
\hline SAMR & $7 / 20$ & 0,72 & $27 / 0$ & $<0,001$ & $9 / 18$ & 0,6 & $8 / 19$ & 0,4 \\
\hline$P$. aeruginosa & $1 / 8$ & 0,018 & $9 / 0$ & 0,078 & $6 / 3$ & $<0,001$ & $5 / 4$ & 0,01 \\
\hline Acinetobacter sp & $3 / 15$ & 0,015 & $18 / 0$ & $<0,001$ & $7 / 11$ & 0,5 & $6 / 12$ & 0,6 \\
\hline Polimicrobiana & $6 / 16$ & 0,70 & $1 / 21$ & 0,90 & $9 / 13$ & 0,6 & $7 / 15$ & 0,4 \\
\hline
\end{tabular}

\begin{tabular}{|c|c|}
\hline Microorganismo & $\mathbf{n}$ \\
\hline Enterococcus sp & 6 \\
\hline Staphylococcus coagulasa negativa & 3 \\
\hline Pseudomonas aeruginosa & 9 \\
\hline SAMR & 6 \\
\hline Acinetobacter $\mathrm{sp}$ & 9 \\
\hline Enterobacter cloacae & 5 \\
\hline Klebsiella pneumoniae & 6 \\
\hline Escherichia coli & 2 \\
\hline Serratia marcescens & 1 \\
\hline Total & 47 \\
\hline
\end{tabular}

El aislado de patógenos multiresistentes (PMR), fue un hecho frecuente, ocurriendo en 94\% (45/48) de los casos, ya sea como cultivo puro o en infecciones polimicrobianas, sin diferencias entre NAVM precoz y tardía (Tabla 2).

El 35\% (17/48) de los pacientes recibió tratamiento antimicrobiano empírico inadecuado, esto se asoció en forma significativa a la presencia de PMR $(p=0,005)$.

La letalidad de la serie fue de $35 \%$ (17/48) y se asoció significativamente al aislado de $P$. aeruginosa $(\mathrm{p}=0,01)$, cuya letalidad específica fue de $56 \%$.

En la Tabla 3 se aprecian diferentes variables clínicas relacionadas con la etiología. El aislado de Acinetobacter sp y $P$. aeruginosa en los cultivos fue significativamente más frecuente en la neumonías de inicio tardío; asimismo no se aislaron cepas de SAMR o Acinetobacter sp si el paciente no había estado expuesto previamente a antimicrobianos. El tratamien- to antimicrobiano inadecuado se asoció en forma significativa a la presencia de $P$. aeruginosa. Sólo la ausencia de co-morbilidad neurológica se asoció significativamente al aislado de Acinetobacter sp ( $<<0,001)$. En la Tabla 4 se señalan las bacterias aisladas en la UCI, de origen no respiratorio, cultivadas durante el período de estudio, con una distribución diferente a la observada en esta serie.

\section{Discusión}

Los principales hallazgos de este estudio se resumen en:

- Staphylococcus aureus resistente a meticilina constituyó el agente etiológico de NAVM más frecuentemente cultivado, ya fuera como agente único o en infecciones polimicrobianas, y tanto en la forma precoz como tardía.

- La recuperación de especies microbianas multiresistentes fue un factor de riesgo para que el tratamiento antimicrobiano resultara inadecuado.

- La presencia de Acinetobacter sp y P. aeruginosa solió ocurrir en las NAVM tardías independientemente de la co-morbilidad asociada.

- Pseudomonas aeruginosa se asoció en forma significativa a una mayor letalidad.

- Finalmente, la ausencia de antimicrobianos previo al diagnóstico de NAVM hizo improbable que se aislara SAMR o Acinetobacter sp.

La mayoría de los estudios publicados sobre etiología de la NAVM señalan a los bacilos gramnegativos como las principales causas, en particular las especies no fermentadoras ${ }^{1,2}$, relegando a un segundo o tercer puesto a SAMR. Por ejemplo, en una extensa revisión publicada por Chastre et $\mathrm{al}^{2}$, los bacilos gramnegativos constituyeron, en promedio, $58 \%$ de la neumonías; en cambio, la suma de SAMR y $S$. aureus meticilina sensible contribuyeron con el $20 \%$ de las causas. Sólo 
George et al en su serie de 26 pacientes coincidió con nuestros hallazgos, pero no tuvo ningún caso de SAMR en NAVM precoz ${ }^{10}$. Diferencias en los hallazgos etiológicos según el área geográfica estudiada no es algo nuevo; de hecho, se sabe que la presencia de cepas de Acinetobacter sp es más frecuente en Europa que en los EUA ${ }^{1}$. Diferencias ocurren también dentro de un país y posiblemente entre hospitales ${ }^{11}$.

Un hallazgo interesante es que la presencia de SAMR ocurrió independientemente de si la neumonía era precoz o tardía, coincidente con lo publicado por Ibrahim et al, quien comparó la etiología de 235 neumonías precoces con 185 tardías sin demostrar diferencias significativas en los grupos analizados ${ }^{12}$. A nuestro juicio, estos hallazgos se pueden explicar por el alto porcentaje de pacientes que recibieron antimicrobianos previo al diagnóstico de NAVM, lo cual favorece la rápida colonización por PMR. Apoya esta hipótesis el hecho de que ningún paciente sin antimicrobianos previo tuvo SAMR. En efecto, Leroy et al en 37 NAVM por PMR demostró que $89 \%$ de ellos habían recibido antimicrobianos previos $(\mathrm{p}=0,007)^{13}$. Es importante señalar que durante el estudio, SAMR no fue el microorganismo predominantemente aislado de fuentes no respiratorias (Tabla 4).

De acuerdo con lo publicado por la literatura especializada, la cobertura rutinaria de SAMR no está indicada en la NAVM precoz y en la tardía, es discrecional según la información epidemiológica local ${ }^{1,2}$. Creemos que en nuestro hospital, una cobertura antimicrobiana empírica en un paciente que los ha recibido anteriormente, debe considerar el SAMR, independientemente del momento del diagnóstico. Esta medida busca reducir la posibilidad de un tratamiento antimicrobiano inadecuado (que en nuestra serie alcanzó a 35\%) dado, principalmente, por la presencia de PMR. Una vez recibido los resultados microbiológicos, si corresponde, se puede reducir el espectro antibacteriano.

A diferencia de lo descrito para SAMR, la presencia de Acinetobacter sp y $P$. aeruginosa predominó en la NAVM tardía, asemejando a lo publicado en las guías de la American Thoracic Society ${ }^{1}$. No obstante, hubo 3 casos de infección por Acinetobacter sp y un caso de infección por $P$. aeruginosa en las NAVM de instalación precoz, de modo que la presencia de PMR no puede descartarse en estas circunstancias. En este sentido, podría ser más apropiado considerar precoz los primeros cuatro días de hospitalización y no de ingreso a la UCI como se recomienda ${ }^{1}$, ya que al menos en nuestra serie hubo una diferencia importante entre las horas transcurridas entre la hospitalización e ingreso a la UCI para la NAVM precoz (Tabla 1).

La letalidad observada fue similar a la esperada por APACHE II de ingreso a la UCI, cuya letalidad calcu- lada fue de $40 \%$; hallazgos similares suelen observarse en pacientes particularmente graves, en los que es difícil demostrar factores que aumenten la ya abultada letalidad de ingreso, como es el caso de pacientes politraumatizados o con distress respiratorio agudo en quienes no hay un aumento significativo en la letalidad atribuible a la NAVM ${ }^{2}$. El exceso de letalidad atribuible a la presencia de cepas de $P$. aeruginosa observado en nuestra serie ha sido descrito con anterioridad, con letalidad superior a $70 \%$ en la mayoría de las publicaciones $^{2}$. En consecuencia, nos parece que este microorganismo debe ser siempre considerado en los tratamientos antimicrobianos empíricos de la NAVM tardía $y$, posiblemente, en la forma precoz que ha recibido tratamiento antimicrobiano previo.

Este trabajo tiene limitaciones a considerar, en primer lugar el número de pacientes puede ser algo reducido; no obstante, en nuestro conocimiento se trata de la mayor serie nacional publicada. El alto porcentaje de pacientes con antimicrobianos previos puede ser un factor que condicione los hallazgos etiológicos; sin embargo, se trata de un hecho real que acontece en la mayoría de las unidades de pacientes críticos y posiblemente nos habla de la necesidad de implementar políticas de control en la prescripción y duración de los tratamientos anti-infecciosos. Otra limitación importante es que los datos obtenidos, por provenir de sólo un centro hospitalario, no pueden ser extrapolados a otros centros nacionales; en consecuencia, esta información debe ser complementada con la generada en otros centros hospitalarios del país, a fin de tener un panorama global acerca de la etiología de la NAVM y los factores que la determinan.

En conclusión, las causas más frecuentes de NAVM en nuestro hospital son SAMR y la etiología polimicrobiana, en forma independiente del momento de su ocurrencia y de existencia de co-morbilidad asociada.

\section{Resumen}

La neumonía asociada a la ventilación mecánica (NAVM) es una complicación relacionada con un aumento de morbilidad y mortalidad. Dentro de los factores de mal pronóstico, el tratamiento antimicrobiano inadecuado es una de las variables que puede corregirse. Para esto debe conocerse la etiología institucional de la NAVM. Durante un año se recopilaron las NAVM con documentación microbiológica en un hospital universitario. En total, 48 neumonías fueron incluidas, 19 en pacientes femeninas, la mediana de la edad fue de 59,5 años (rango 17-91), 12 de ellas precoces. Staphylococcus aureus meticilina resistente (MRSA) fue el principal agente involucrado, independiente del mo- 
mento de su génesis, seguido por la etiología polimicrobiana, Acinetobacter sp y Pseudomonas aeruginosa, en ese orden. La etiología no se asoció con la existencia de co-morbilidad, el uso previo de antimicrobianos se asoció con la presencia de MRSA y etiología polimicrobiana. La letalidad fue de $35 \%$ y se relacionó, principalmente, con la presencia de $P$. aeruginosa. Conclusión: La principal causa de NAVM en esta experiencia fue SAMR, independiente del momento evolutivo de su ocurrencia.

\section{Referencias}

1.- Niederman M S, Craven D E. Guidelines for the management of adults with hospitalacquired, ventilator-associated, and healthcare-associated pneumonia. Am J Respir Crit Care Med 2005; 171: 388-416.

2.- Chastre J, Fagon J Y. Ventilator-associated pneumonia. Am J Respir Crit Care Med 2002; 165: 867-903.

3.- Jiménez $\mathrm{P}$, Torres $\mathrm{A}$, Rodríguez-Roisin $\mathrm{R}$, de la Bellacas J P, Aznar R, Gatell J M, et al. Incidence and etiology of pneumonia acquired during mechanical ventilation. Crit Care Med 1989; 17: 882-5.

4.- Baker A M, Meredith J W, Haponik E F. Pneumonia in intubated trauma patients. Microbiology and outcomes. Am J Respir Crit Care Med 1996; 153: 343-9.

5.- Iregui M, Ward S, Sherman G, Fraser V J, Kollef M H. Clinical importance of delays in the initiation of appropriate antibiotic treatment for ventilator-associated pneumonia. Chest 2002; 122: 262-8

6.- Torres A, Aznar R, Gatell J M, Jiménez P, González J, Ferrer A et al. Incidence, risk and prognosis factors of nosocomial pneumonia in mechanically ventilated patients. Am Rev Respir Dis 1990; 142: 523-8

7.- Arancibia F, Fica A, Hervé B, Ruiz M, Yunge M. Diagnóstico de neumonía asociada a ventilación mecánica. Rev Chil Infectol 2001, vol 18 supl 2, p 41-57.

8.- Rello J, Paiva J A, Baraibar J, Barcenilla F, Bodi $\mathrm{M}$, Castander D, et al. International conference for the development of consensus on the diagnosis and treatment of ventilator-associated pneumonia. Chest 2001; 120: 955-70.

9.- Knaus W A, Draper E A, Wagner D P, Zimmerman J E. APACHE II: a severity of disease classification system. Crit Care Med 1985; 13: 818-29.

10.- George D L, Falk P S, Wunderink R G,
Leeper K V, Meduri G U, Steere E L, et al. Epidemiology of ventilator-acquired pneumonia based on protected bronchoscopic sampling. Am J Respir Crit Care Med 1998; 158: 1839-47.

11.- Rello J, Sa-Borges M, Correa H, Leal S R, Baraibar J. Variation in etiology of ventilator-associated pneumonia across four treatment sites. Implications for antimicrobial prescribing practices. Am J Respir Crit Care Med 1999; 160: 608-13.

12.- Ibrahim E H, Ward S, Shermann G, Kollef M H. A comparative analysis of patients with early-onset vs late-onset nosocomial pneumonia in the ICU setting. Chest 2000; 11: 1434-42.

13.- Leroy O, Jaffré $S$, d'Escrivan T, Devos P, Goerges H, Alfandari S, et al. Hospitalacquired pneumonia. Risk factors for antimicrobial-resistant causative pathogens in critically ill patients. Chest 2003; 123 : 2034-42. 